\title{
Comparison of Terahertz Pulse Imaging and Near-Infrared Spectroscopy for Rapid, Non-Destructive Analysis of Tablet Coating Thickness and Uniformity
}

\author{
Robert P. Cogdill • Ryanne N. Forcht • Yaochun Shen • \\ Philip F. Taday • J. Richard Creekmore • \\ Carl A. Anderson • James K. Drennen III
}

Published online: 16 October 2007

(C) International Society for Pharmaceutical Engineering 2007

\begin{abstract}
In this study, coating thickness and uniformity of production-scale pharmaceutical tablets were investigated using near-infrared (NIR) and terahertz pulse imaging (TPI) spectroscopy. Two coating formulations were considered; samples for each coating formulation were obtained at 0,1 , $2,3,4$, and $5 \%$ coating weight. NIR spectra were collected, and regressed with respect to batch percent weight gain. While standard errors of calibration (SEC) less than $0.5 \%$ were observed for both formulations, the calibrations were not specifically sensitive to coating thickness. An upper limit for NIR coating thickness analysis was estimated to be $\sim 4$ $6 \%$ weight gain for this system. The NIR calibrations were used as filters to choose subsets of samples for TPI, and as a secondary method for validation of TPI results. The features in TPS time-domain spectra result when an incident $\mathrm{THz}$ plane wave meets a refractive index interface, which may be converted to an absolute distance. Therefore, assuming that a discernible difference in refractive index between coating material and core exists, coating thickness can be determined
\end{abstract}

\section{R. P. Cogdill}

Duquesne University Center for Pharmaceutical Technology,

410A Mellon Hall, 600 Forbes Ave.,

Pittsburgh, PA 15282, USA

R. N. Forcht • C. A. Anderson • J. K. Drennen III $(\varangle)$

Duquesne University Graduate School of Pharmaceutical Sciences,

410A Mellon Hall, 600 Forbes Ave.,

Pittsburgh, PA 15282, USA

e-mail: drennen@duq.edu

Y. Shen • P. F. Taday

TeraView, Ltd., Cambridge, UK

J. R. Creekmore

AstraZeneca, LP, Wilmington, DE, USA non-destructively. Coating thickness measurements from TPI and NIR spectroscopy were compared to estimate the lower limit for quantitative TPI coating analysis; a lower limit of $\sim 35 \mathrm{~mm}$ was obtained for this system. Optical microscopy was employed on a subset of samples to validate absolute thickness values; reasonable correlations between the three methods were obtained. TPI was considered advantageous relative to the other methods, as similar results were obtained without the need for destructive sampling or empirical calibration development.

Keywords Tablet coating · Thickness · Terahertz - Imaging · Spectroscopy $\cdot$ Near-infrared $\cdot$ Chemometrics $\cdot$ PAT

\section{Introduction}

For many drug products administered in solid dosage form, tablet coating thickness and uniformity are important critical-to-quality (CTQ) attributes of the final product. Indeed, coating appearance is likely the most tangible aspect of tablets from which patients derive perceptions of product quality. In addition to determining the aesthetic qualities of finished dosage forms, tablet coatings maintain product stability by providing a protective barrier against the destructive influences of atmospheric oxygen, ultraviolet radiation, or humidity [1]. Following oral administration, functional tablet coatings may be used to conceal the bitter, salty, or offensive taste of a drug substance, or to protect a drug substance from the destructive influence of gastric acid during transit to the site of absorption [1].

As drug substances and dosing regimens have become more complex, functional polymeric tablet coatings are 
increasingly being used as a means for attaining "programmable" control over the rate of disintegration, dissolution, and, hence, bioavailability of certain drugs. If coatings are non-uniform or contain surface defects, the desired rate of dose delivery and total bioavailability may be compromised. These issues may arise from problems with the coating pan or spray processes or from flaws in the coating materials. Since the assurance of uniform bioavailability of drug products is among the top concerns of both the industry and the U.S. Food and Drug Administration, drug product disintegration and dissolution testing is required for product release into the supply chain. For all of these reasons, effective monitoring and control of tablet coating operations is critical to maintaining process capability and efficiency.

High-speed process analytical sensors (e.g. PAT) [2-5] capable of quickly and accurately determining coating depth or thickness are essential for control of, and real-time release from, tablet coating operations. Many analytical techniques have been used for analysis of tablet coatings, including optical microscopy, atomic force microscopy, confocal laser microscopy, X-ray photoelectron spectroscopy, electron paramagnetic resonance, Fourier transform infrared spectroscopy, scanning thermal microscopy [6], and laserinduced breakdown spectroscopy [7]. While these methods are generally very powerful, they are less than ideal for real-time process analytics since they are all either destructive or cannot be readily implemented for rapid online measurement [6]. Near-infrared (NIR) absorbance spectroscopy [8] and time-domain terahertz pulse imaging (TPI) $[6,9]$ have been demonstrated to be useful for rapid, nondestructive analysis of tablet coating thickness.

Near-infrared spectrometers are one of the most powerful and versatile types of instrumentation used for process analysis of pharmaceutical solids. There are few operations employed during the manufacture of solid dosage tablets for which NIR spectroscopic monitoring does not offer benefits in terms of process understanding and/or control [8]. Previous experiments have shown that NIR spectroscopy can be used for single-tablet coating thickness analysis and can be correlated to drug release [10]; another group has shown that non-contact NIR reflectance spectroscopy can be used for real-time control of some tablet coating operations [11]. While such results are promising, both experiments illustrate two major difficulties and limitations of using NIR spectroscopy for analysis of tablet coatings.

First, NIR coating measurements are highly empirical. While certain coating materials have unique NIR signatures that become more intense as coating thickness increases, the most prominent features correlated with increasing coating thickness are related to the relative "disappearance" of the tablet core from the NIR spectrum. Though such signals can be analytically useful, they are inherently indirect and, hence, their specificity is limited to the caveat that the spectral signature of the tablet core does not vary significantly. Second, since NIR measurement of coating thickness is indirect, there exists for every coated tablet a unique limit after which the NIR signal will no longer be sensitive to increasing coating depth. For highly opaque coatings, such as those with a high concentration of titanium dioxide, $\mathrm{TiO}_{2}$, or for coatings with high specular reflectivity, the thickness limit for accurate prediction of coating thickness via NIR spectroscopy can be quite variable.

Terahertz pulse imaging spectroscopy addresses the limitations of NIR for analysis of tablet coating thickness and uniformity. Terahertz pulse imaging is a nondestructive pharmaceutical analysis tool using low power, ultrashort pulses of electromagnetic radiation at lower frequencies than traditional infrared techniques [9]. Terahertz (THz) spectroscopy, in general, covers the spectral range from about $3 \mathrm{~cm}^{-1}$ to about $600 \mathrm{~cm}^{-1}$, also known as the farinfrared (far-IR) region of the electromagnetic spectrum, implying frequencies on the order of approximately 0.1 to $20 \mathrm{THz}$, as $1 \mathrm{THz}$ is equivalent to $33 \mathrm{~cm}^{-1}[9,12,13]$.

Terahertz time-domain spectroscopy, on which the fundamentals of TPI are based, implies generation and detection of $\mathrm{THz}$ pulses in a synchronous, coherent manner using visible or NIR laser pulses. Pulse widths range from approximately $100 \mathrm{fs}$ down to about $10 \mathrm{fs}$. Hence, using $\mathrm{THz}$ time-domain spectroscopy, it is now possible to carry out time-resolved far-IR studies with sub-picosecond temporal resolution [12]. Because of the optical gating technology used, TPI can achieve a very high signal-tonoise ratio $\left(>10^{5}\right)$. As a coherent technique, both the transient electric field amplitude and phase information of $\mathrm{THz}$ pulses are measured simultaneously, which allows the frequency-domain spectral absorption, $\alpha(\omega)$, and refractive index, $n(\omega)$, coefficients of the material under investigation to be extracted from a single measurement [14]. In-depth description of the theory, instrumentation, and application of $\mathrm{THz}$ pulse spectroscopy has been provided by numerous earlier studies and reviews [9, 12-14]. Recent studies utilizing frequency-domain analysis of $\mathrm{THz}$ time-domain spectra have shown that, while it is at a relatively early stage of development, $\mathrm{THz}$ pulse spectroscopy has great potential as a quantitative spectroscopic method for analysis of pharmaceutical solids complimentary to such methods as NIR [12, 15-18].

Without transformation to the frequency-domain, timedomain TPI signals can be used for direct determination of the thickness of layered materials such as tablet coatings. Time-domain $\mathrm{THz}$ spectra are generated by measuring the intensity of $\mathrm{THz}$ reflections over a range of probe beam delays [6]. The features in TPS time-domain spectra correspond to relatively intense reflection events that occur when an incident $\mathrm{THz}$ plane wave meets a refractive index interface (Fig. 1). Typically, a positive peak indicates a 


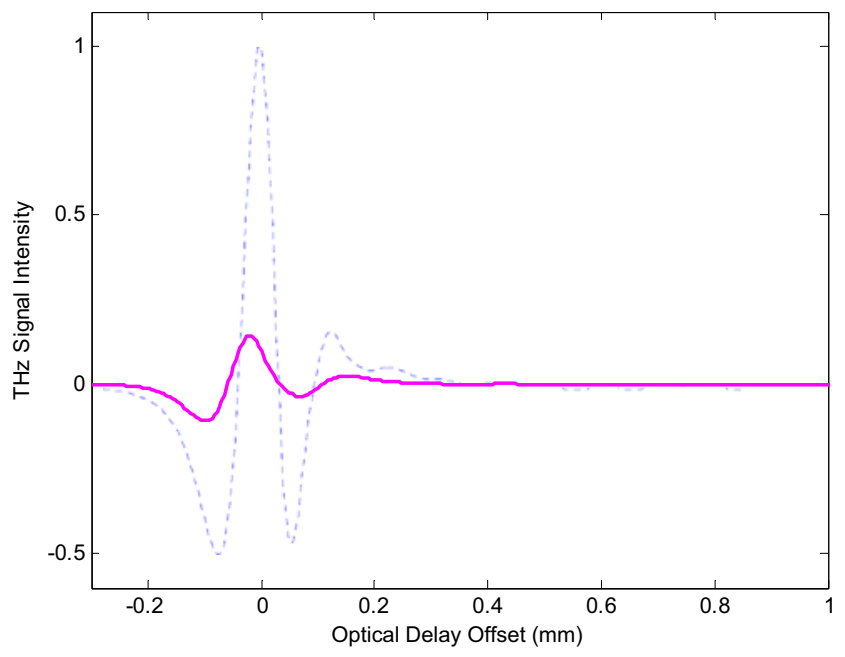

Fig. 1 Raw (no preprocessing) terahertz time-domain intensity scan of a coated tablet from the $5 \%$ weight gain batch. The dashed line illustrates the mirror reference pulse intensity, while the solid line is the intensity of light reflected from the tablet over a range of optical delay. Note that the horizontal axis describes the distance traveled by the optical delay line, and is proportional to photon time of flight

transition from lower to higher refractive index [19]. If refractive index is known, or if it can be estimated with reasonable accuracy, the time delay of the probe beam can be converted to distance, or layer thickness.

The time-domain TPS signal can be considered analogous to an ultrasound B-scan, whereby the depth and thickness of interfaces can be estimated with finite accuracy. By raster-scanning across the surface of a sample, a two-dimensional map or image of TPS signals can be generated. Since most tablet coating materials are semitransparent in the $\mathrm{THz}$ region of the electromagnetic spectrum, it is anticipated TPI can be used to generate accurate images of thickness for relatively thick coatings. Furthermore, since tablet coating materials have relatively stable refractive indices, which can easily be determined a priori, TPI measurements are expected to be useful as a primary method for nondestructive coating thickness measurement, requiring little or no calibration for a wide variety of coatings and substrates. The lower-limit of TPI coating thickness analysis is known to be dependent on the width of THz pulses used to generate TPI waveforms, and the strength of refractive index interface between the tablet coating and core.

\section{Objectives}

The objectives of this work were to:

1) Demonstrate the use of terahertz pulse imaging for the nondestructive analysis of coating thickness for production-scale solid dosage tablets.
2) Determine the lower limit of accurate terahertz coating thickness measurement.

3) Compare the relative utility of terahertz pulse imaging and NIR spectroscopy for tablet coating analysis.

\section{Experimental}

\section{Materials}

The objectives of this research were achieved by analyzing a series of production-scale solid dosage tablets having coating thickness spanning a range of designed variation. Two coating formulations were considered for this work: Opadry White and Opadry II Pink. During the tablet coating process, samples of tablets were withdrawn according to aggregate percent weight gain; an excess of samples were collected at levels of $0,1,2,3,4$, and $5 \%$ nominal weight gain. The tablet cores were produced and coated at an industrial research facility; further details on the cores and coating procedure were not available during this work.

\section{Data Acquisition, Instrumentation, and Software}

Near-infrared single-point reflectance spectra were collected for a large subset of the available tablets at each coating level and for each coating formulation using a FOSS NIRSystems 5000 spectrometer (Silver Spring, MD). The NIR spectra spanned the range from 1,098-2,498 nm, with a 2-nm wavelength increment. The spectral data were regressed with respect to designed percent weight gain to generate a calibration model to predict pseudo-weight gain, which is expected to be highly correlated to coating thickness. This method was used as a secondary method for validation of TPI results, and was also used as a filter to choose a smaller subset of samples for TPI. Samples having especially large or small prediction residuals with regard to the pseudo-weight gain calibration model were chosen over the entire range of predicted coating weight; subsets of 21 and 16 tablets were selected for the white and pink coating formulations, respectively.

A THz scan from both sides of each selected tablet was acquired in reflectance mode using a TPI Coating Scan (TeraView Limited, Cambridge, UK). Sharp curvature of the tablets near the equatorial edge of the samples was expected to create adverse scattering effects; therefore, images were limited to a $7.0 \mathrm{~mm}$ diameter spot near the center of each tablet. An image of one side of each tablet was acquired within approximately $7 \mathrm{~min}$ of scan time. Scanning parameters were held constant for all tablets analyzed.

The deconvolution of time-domain TPI waveforms and generation of coating analysis parameters and outputs were 
handled by batch software provided by TeraView. Details of the deconvolution procedure are described in earlier studies $[20,21]$. Chemometric and statistical analyses of the data were performed in the MATLAB programming environment (v7.1, The MathWorks, Natick, MA) using PLS Toolbox (v3.0, Eigenvector Research, Manson, WA), along with many analysis routines developed in-house for this work (Duquesne University Center for Pharmaceutical Technology-DCPT, Pittsburgh, PA).

Optical microscopy was performed as an independent reference method for a subset of tablets using a Leica S6D StereoZoom (Wetzlar, Germany) with integrated SPOT Insight color digital camera (California, USA). Samples were bisected along the diameter using a fine blade microtome then placed on a slide under the microscope such that the sliced face was perpendicular to the camera lens. Images were collected at $40 \times$ magnification and were viewed with SPOT Insight Basic software package (California, USA). Digital images were acquired having 7.68 megapixels $(3,200 \times 2,400$ pixels $)$; actual pixel size $(\sim 0.92 \mu \mathrm{m} / \mathrm{pixel})$ and spatial resolution $(\sim 2.76-3.1 \mu \mathrm{m})$ were calibrated using a USAF 1951 metal foil resolution target. The mean and standard deviation of coating thickness for each tablet fragment were determined by manually measuring the pixel width at 30 locations within each image. Manual image analyses were replicated by three analysts working independently to mitigate the effect of personal bias.

\section{Results and Discussion}

\section{NIR Spectroscopy}

The mean NIR spectrum for each level of coating weight, grouped according to coating formulation, is shown in Fig. 2. The trends in relative NIR absorbance intensity agree with observations from earlier research, whereby increasing coating weight (thickness) was correlated to decreasing absorbance intensity of spectral features related to the tablet core [10]. The only spectral feature for either formulation which may have positive correlation to coating weight is in the region of $\sim 2,280 \mathrm{~nm}$; while the intensity of the band did not increase with increasing coating weight, the rate of intensity decrease was much lower. The location of the spectral feature suggests that it may be related to a small $\mathrm{H}_{2} 0$ combination band, or to a much broader region of $\mathrm{CH}_{\mathrm{n}}$-combination bands, which would be logical considering the polymeric base for the coating formulations.

Another interesting feature of the NIR spectra is the reduction in the rate of absorbance attenuation as the coating level increases. The relative spectral change between 0 and

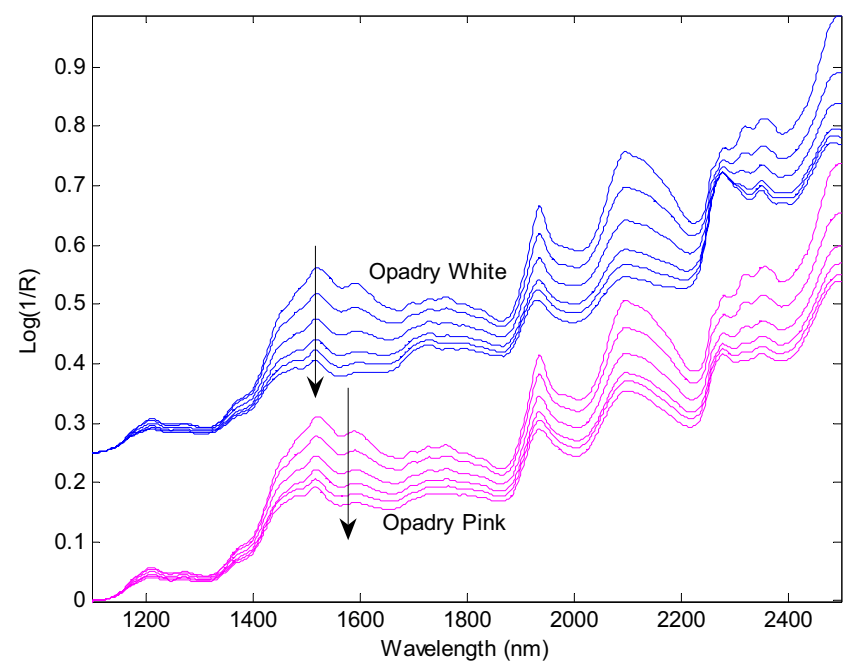

Fig. 2 Average NIR spectrum for each coating batch $(0,1,2,3,4$, and 5\% coating weight). The arrows indicate the direction of intensity change with increasing coating weight. Each spectrum has been offset by subtracting its intensity at $1,098 \mathrm{~nm}$; furthermore, a constant offset has been added to the Opadry White scans for illustrative purposes

$1 \%$, or 1 and $2 \%$ coating weight is apparently much greater than the spectral change between 4 and $5 \%$ coating weight. The results for polynomial regression of NIR absorbance intensity at absorbance peaks against coating weight gain (not shown) suggests that, if the observed trends held, the saturation limit of the NIR signal would be reached between 5 and $6 \%$ weight gain across most of the spectrum (some of the bands at shorter wavelengths are predicted to saturate in the range between 6 and 7\%).

Partial least-squares (PLS) regression was used to estimate calibration models to predict coating weight for each tablet; the calibration model for both formulations utilized standard normal variate (SNV) preprocessing with a single PLS factor [8]. The coefficients of determination $\left(R^{2}\right)$ for Opadry White and Opadry II Pink were 0.97 $\left(n_{\text {scans }}=197\right)$ and $0.93\left(n_{\text {scans }}=354\right)$, respectively (Note: two scans per one tablet). Approximately twice as many Opadry II Pink tablets were analyzed since samples were supplied from two batches; no significant spectral differences between batches were observed. Observed standard errors of calibration (SEC) were 0.32 and $0.42 \%$ for Opadry White and Opadry II Pink, respectively. The fit of the calibration models was very good considering that batchlevel weight gain was used for individual tablet reference measurements, which is expected to have finite error.

The trends in predicted values shown in Fig. 3 demonstrate that the calibration is quite linear up to $4 \%$ weight gain, after which it appears to taper towards a negative bias. This supports the notion that the saturation limit for NIR analysis of coating thickness (for these tablets) is in the range of $5-7 \%$ coating weight. Furthermore, the use of more opaque coating materials (e.g. higher 


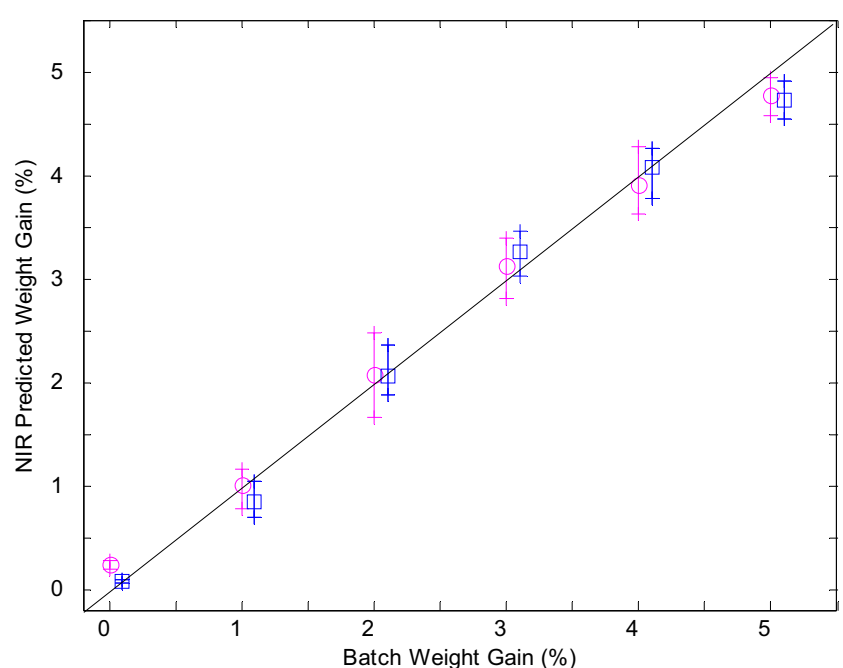

Fig. 3 Correlation between batch reference percent weight gain and NIR estimated percent weight gain for Opadry White (squares), and Opadry Pink (circles). Each point indicates the median predicted value, while the vertices of the error bars extend to the 25 and $75 \%$ quartiles. A small offset has been added to the Opadry White reference data for illustrative purposes

$\mathrm{TiO}_{2}$ content), or tablet cores with less intense absorption features, may drastically decrease the expected saturation limit. Finally, the effect of variation in the spectral profile of the tablet cores (e.g. concentration, process signature [3]) is not known. Since the spectral signature related to coating thickness is highly non-specific, a much more in-depth experimental design would likely be necessary to generate a multivariate calibration model suitably robust to be deployed for in-process coating measurement. With this in mind, the value of a more direct, selective method of analysis is obvious.

\section{Time-Domain $\mathrm{THz}$}

The mean time-domain $\mathrm{THz}$ spectrum at each coating level for the Opadry II Pink samples is shown in Fig. 4, with a magnified view of the waveforms in Fig. 5. The largest feature in the waveforms (at zero optical delay) is related to the refractive index change at the air-tablet interface. The relative difference in optical delay between the air-tablet interface and the next largest feature in the waveform is used (in conjunction with expected refractive index) to predict coating thickness with spatial resolution (limited by diffraction) on the order of $\sim 200 \mu \mathrm{m}$ (Fig. 6).

In contrast to trends in NIR signal, with increasing coating thickness, the relative magnitude of distance contrast seems to become more distinct between 4 and $5 \%$ weight gain. This suggests both that TPI analysis is capable of measuring coating thickness to greater depth, and that there is a definite lower-limit for quantitative prediction of coating thickness. In this regard, it would

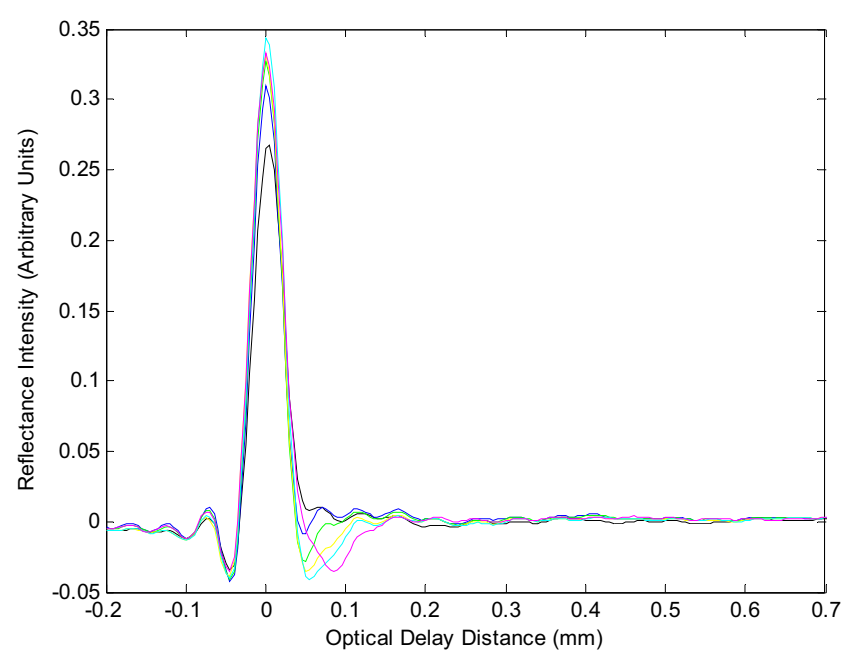

Fig. 4 Deconvolved time-domain $\mathrm{THz}$ spectra from Opadry Pink coated tablets. Each trace is the average of scans from multiple tablets of the same reference (\%) weight gain. All sample waveforms are offset during the deconvolution and coating analysis procedure so that the center of the largest peak, which corresponds to the air-tablet interface reflection event, is relocated to coincide with the zero time delay point

seem that TPI and NIR spectroscopy are complementary tools for coating thickness determination.

Correlation of TPI coating thickness and optical microscopy measurements for selected samples is shown in Fig. 7. Ellipses were used to indicate mean and \pm 1 standard deviation in the axis of the corresponding measurement; hence, vertical ellipses indicate greater variance in the TPI measurement, while horizontal ellipses indicate greater variance in the optical microscopy measurement.

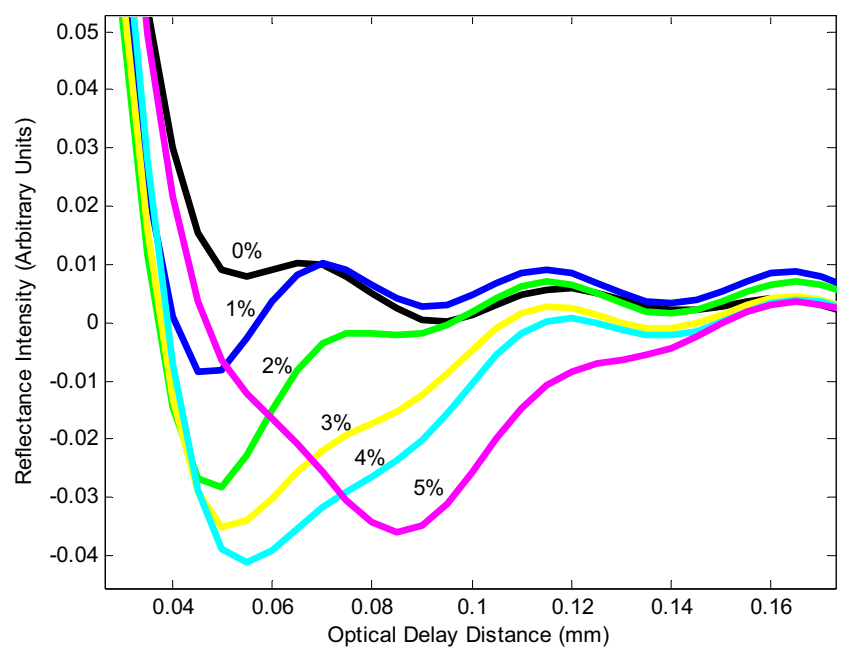

Fig. 5 Expanded view of deconvolved time-domain $\mathrm{THz}$ spectra from Opadry Pink coated tablets. Each trace is the average of scans from multiple tablets of the same reference (\%) weight gain. The TeraView coating analysis algorithm estimates outer coating layer thickness from the dimensions and position of the first significant signal feature after the air-tablet interface 
Fig. 6 Screen shot of coating analysis results for coated tablets having $4 \%$ (a) and 3\% (b) weight gain. The upper panels are false-color illustrations where color intensity is proportional to layer thickness; the lower panels are histogram summaries of layer thickness distribution for each tablet

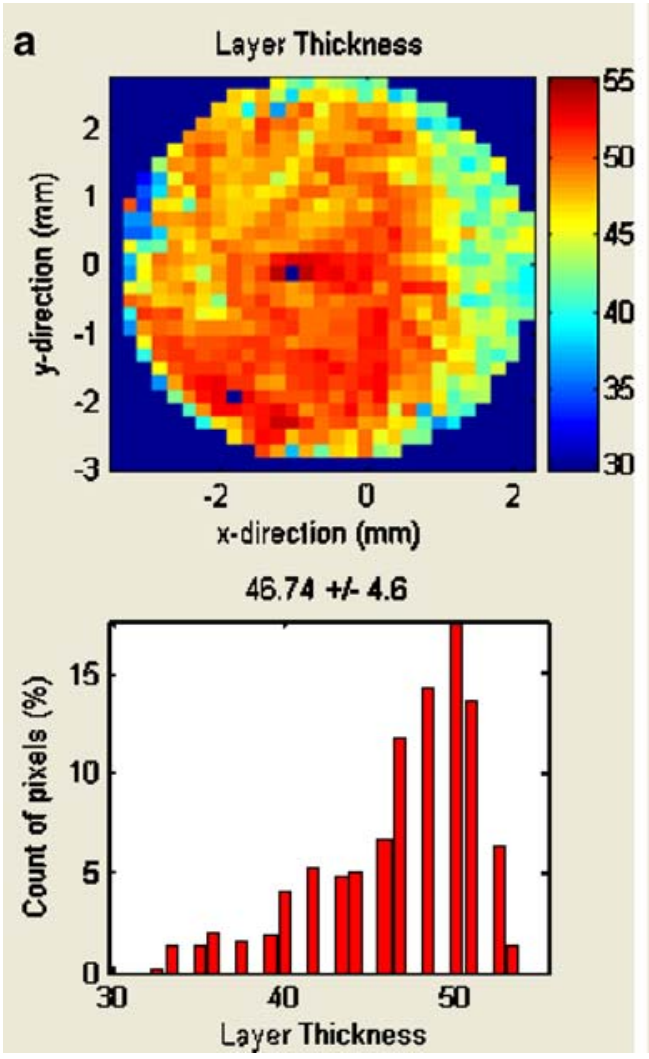

The mean and standard deviation of each TPI measurement was estimated from the individual pixels across surface of the tablet image, while the mean and standard deviation of each optical microscopy measurement is determined for only

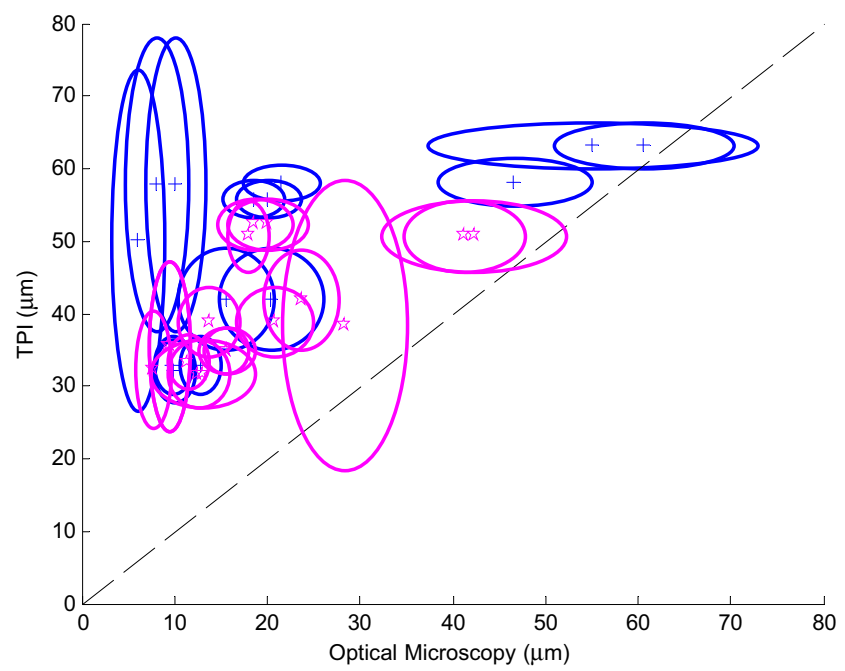

Fig. 7 Correlation between optical microscopy and time-domain $\mathrm{THz}$ layer thickness measurements for Opadry White (crosses) and Opadry Pink (stars). The height of the confidence ellipse surrounding each data point is proportional to the standard deviation of the $\mathrm{THz}$ measurements within a single tablet TPI image, whereas the width of each confidence ellipse is proportional to the standard deviation of layer thickness as determined by optical microscopy a portion of a single equatorial slice of each tablet. While significant error between the TPI and optical microscopy measurements was observed, the fact that TPI is relatively accurate on an absolute basis is encouraging. As was suggested in earlier paragraphs, the TPI coating thickness measurement is apparently more accurate at greater coating thickness levels. Furthermore, it is important to keep in mind that no effort was made to calibrate or optimize the TPI measurements for these samples.

While optical microscopy is utilized as an independent reference method for this work, there is an inherent expectation of some disagreement between microscopy and spectroscopic measurements. First, it is virtually impossible accurately determine the corresponding location of spectroscopic and microscopy measurements. Second, the destructive nature of tablet cleavage, along with the variation in the angle of the slice profile, is expected to add to measurement error in unpredictable ways. Finally, and likely most relevant to comparison with TPI, many tablets were encountered for which the interface between the tablet coating and core was not easily determined by eye, since the coating seemed to penetrate the surface of the tablet core (Fig. 8), which may have added a negative bias to the microscopy measurements (relative to TPI). While the concept of coating "thickness" is more arbitrary in such cases, the repeatability of instrumental techniques such as TPI (relative to manual techniques) improves the objectivity of analyses. 


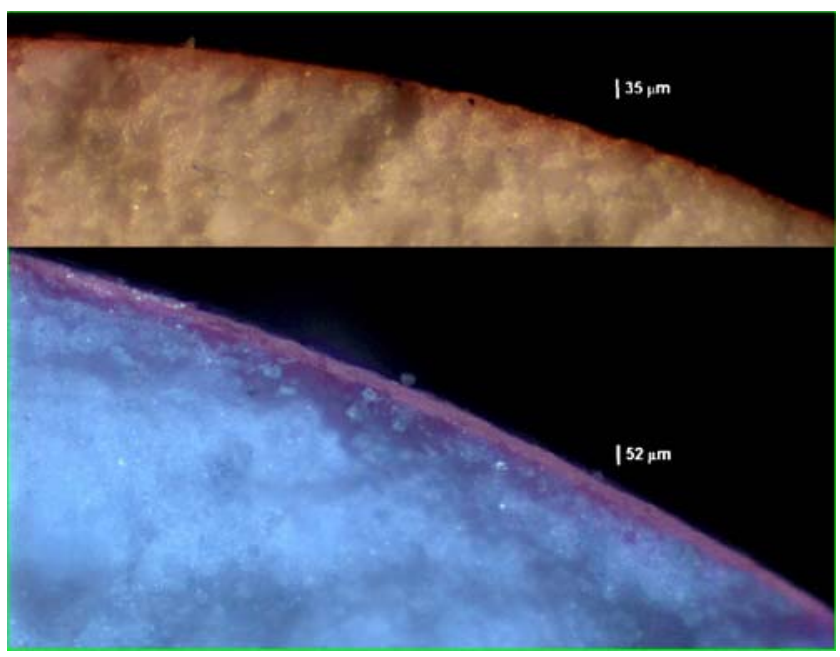

Fig. 8 Example images from the optical microscopy coating layer thickness measurement procedure. The tablet in the upper panel was drawn from a 3\% weight gain batch, while the tablet from the lower panel was drawn from a 5\% weight gain batch. These tablet images illustrate the range in layer quality (sharpness) that was observed, which hindered the ability to conclusively identify layer transitions by visual inspection

Since relatively few tablets were subjected to analysis by optical microscopy, and since such results are expected to be more error-prone, the correlation with NIR spectroscopy was used to estimate the lower limit for accurate TPI analysis. Since positive, linear correlation was observed between NIR predicted weight gain and TPI-predicted coating thickness for tablets with predicted weight gain greater than $\sim 2.75 \%$ (not shown), it was anticipated that TPI could serve as a reference technique to build a NIR calibration for tablet coating thickness on an absolute basis over a limited range.

By subsequently generating predicted coating thickness values for all samples, the limit of TPI measurement

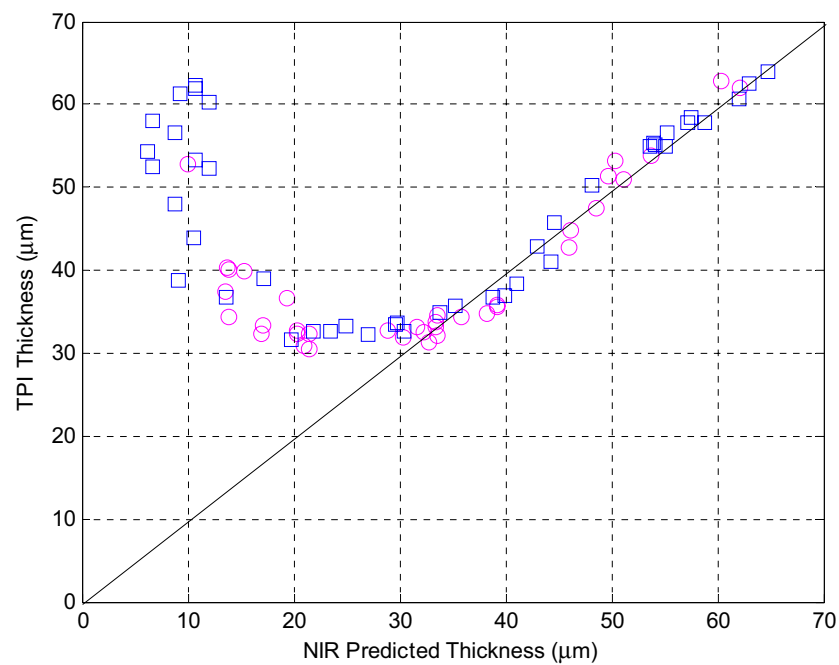

Fig. 9 Correlation between NIR and THz coating analyses for Opadry White (squares) and Opadry Pink (circles)

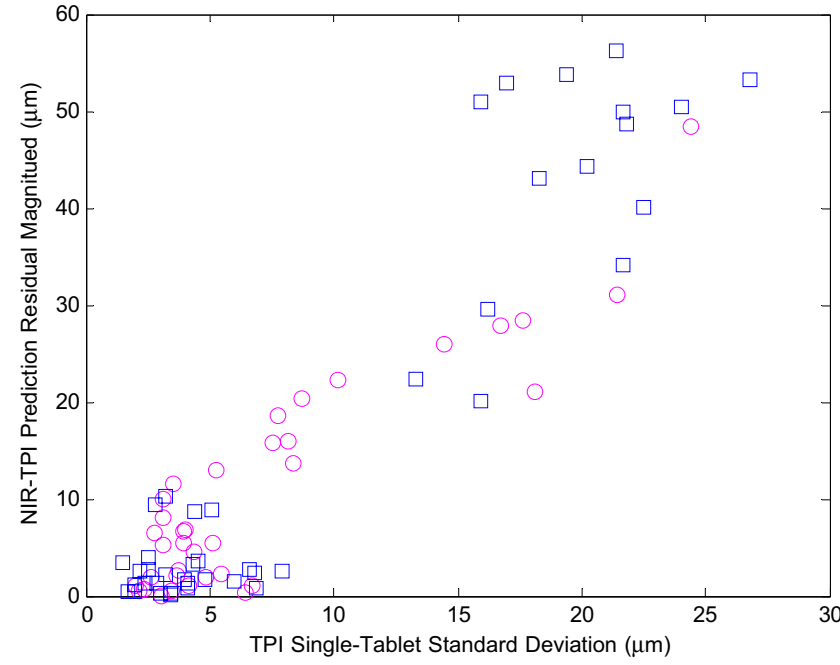

Fig. 10 Correlation between the standard deviation of TPI coating thickness measurements across the surface of each tablet, and the absolute value of residual prediction error between NIR and TPI measurements. The figure illustrates that, as coating layer thickness and TPI measurement quality decreases, the standard deviation of single-pixel coating measurements increases

suitability can be determined as the point at which the linear trend between TPI and NIR is broken (Fig. 9). These results agree with earlier estimations by other researchers that the lower limit for TPI coating thickness analysis (given the current equipment and methods) is approximately $35 \mu \mathrm{m}$.

One troubling aspect of these data, however, is that the TPI results for coating levels beneath the lower measurement limit tend to trend higher. Such measurement errors would be expected to arise when the magnitude of $\mathrm{THz}$ reflections related to the internal microstructure of the tablet core exceeds the magnitude of reflections related to the core-coating interface. It was observed, however, that the estimated standard deviation of coating thickness for such tablets was much larger than for other tablets. Again, this is not unexpected since there were no uniform layers anticipated within the tablet cores. With these results in mind, rules for TPI coating analysis with regard to sample standard deviation might be used to determine the suitability of any individual measurement, as depicted in Fig. 10.

\section{Conclusions}

The objectives of this research were achieved by analyzing production-scale solid dosage tablets having a wide range of designed coating thickness variation. The NIR diffuse reflectance spectroscopy and time-domain TPI data were compared to determine the relative strengths and limitations of each technique.

The results for NIR spectroscopy are in agreement with the findings of earlier researchers who demonstrated that 
NIR is an indirect, non-specific measurement of coating thickness, which is limited by the effective maximum depth interrogated by remitted NIR radiation. Furthermore, as an empirical technique, NIR spectroscopy requires multivariate calibration for quantitative use.

The results for TPI demonstrate that the method is capable of measuring tablet coating thickness and uniformity without the need for multivariate calibration. Comparison of TPI and optical microscopy measurements indicate that, while the TPI measurements were apparently correlated, there is currently a bias and sensitivity difference between the two methods. However, as it is subject human error and bias, optical microscopy is not an optimal reference method. However, by estimating a NIR linear calibration model for coating thickness using TPI as a reference measurement, and subsequently comparing the prediction results, the lower limit for quantitative TPI analysis of coating thickness was determined to be approximately $35 \mu \mathrm{m}$.

In comparison to NIR or other process analytical technologies, terahertz is relatively new. While the performance of current $\mathrm{THz}$ methods for quantitative spectroscopic analysis of pharmaceutical solids and tablet coatings is compelling, there are likely to be many opportunities to improve the technique as the number of feasible applications for $\mathrm{THz}$ increases. Further research using TPI for coating analysis will investigate new materials, opportunities for increasing sensitivity, and comparisons with other imaging modalities.

Acknowledgements The authors would like to thank the Duquesne University department of biology for use of their microscopy facilities and equipment.

\section{References}

1. Ansel HC, Allen LV Jr, Popovich NG. Capsules and tablets. Pharmaceutical dosage forms and drug delivery systems. 7th ed. Philadelphia, PA: Lippincott Williams \& Wilkins; 1999. p. 179-228.

2. Cogdill RP, Anderson CA, Delgado-Lopez M, Chisholm R, Bolton R, Herkert T, et al. Process analytical technology case study, part II: development and validation of quantitative for tablet API content and hardness. AAPS PharmSciTech 2005;6 (2):273-83.

3. Cogdill RP, Anderson CA, Delgado-Lopez M, Molseed D, Chisholm R, Bolton R, et al. Process analytical technology case study, part I: feasibility studies for quantitative NIR method development. AAPS PharmSciTech 2005;6(2):262-72.
4. Cogdill RP, Anderson CA, Drennen JK. Process analytical technology case study, part III: calibration monitoring and transfer. AAPS PharmSciTech 2005;6(2):284-97.

5. FDA. PAT - a framework for innovative manufacturing and quality assurance; 2004

6. Fitzgerald AJ, Cole BE, Taday PF. Nondestructive analysis of tablet coating thickness using terahertz pulsed imaging. J Pharm Sci 2004;94(1):177-83.

7. Mowery MD, Sing R, Kirsch JD, Razaghi A, Béchard S, Reed RA. Rapid at-line analysis of coating thickness and uniformity on tablets using laser induced breakdown spectroscopy. J Pharm Biomed Anal 2002;28:935-43.

8. Cogdill RP, Drennen JK. Near-infrared spectroscopy. In: Brittain HG, editor. Spectroscopy of pharmaceutical solids. New York, NY: Taylor \& Francis Group; 2006. p. 313-412.

9. Taday PF. Applications of terahertz spectroscopy to pharmaceutical sciences. Philos Trans R Soc Lond A 2004;362:351-64.

10. Kirsch JD, Drennen JK. Determination of film-coated tablet parameters by near-infrared spectroscopy. J Pharm Biomed Anal $1995 ; 13: 1273-81$.

11. Pérez-Ramos JD, Findlay WP, Peck GE, Morris KR. Quantitative analysis of film coating in a pan coater based on in-line sensor measurements. AAPS Pharm Sci Techol 2005;6(1):E127-36.

12. Beard MC, Turner GM, Schumuttenmaer CA. Terahertz spectroscopy. J Phys Chem B 2002;106:7146-59.

13. Hangyo M, Tani M, Nagashima T. Terahertz time-domain spectroscopy of solids: a review. Int J Infrared Millim Waves 2005;26(12):1661-90.

14. Lucarini V, Ino $\mathrm{Y}$, Peiponen K-E, Kuwata-Gonokami M. Detection and correction of the misplacement error in terahertz spectroscopy by application of singly subtractive Kramers-Kronig relations. Phys Rev, B 2005;72(12):125107(125106).

15. Cogdill RP, Short SM, Forcht RN, Shi Z, Shen YC, Taday PF, et al. An efficient method development strategy for quantitative chemical imaging via terahertz pulse spectroscopy. J Pharm Innovation 2006; 1(1):63-75.

16. Shen YC, Lo T, Taday PF, Cole BE, Tribe WR, Kemp MC. Detection and identification of explosives using terahertz pulsed spectroscopic imaging. Appl Phys Lett 2005;86(241116):24-31.

17. Shen YC, Taday PF, Newnham DA, Kemp MC, Pepper M. 3D Chemical mapping using terahertz pulse imaging. Proc SPIE 2005;5727:24-31.

18. Strachan CJ, Rades T, Newnham DA, Gordon KC, Pepper M, Taday PF. Using terahertz pulsed spectroscopy to study crystallinity of pharmaceutical materials. Chem Phys Lett 2004;290: $20-4$.

19. Neil GR, Carr GL, Gubeli JF III, Jordan K, Martin MC, McKinney WR, et al. Production of high power femtosecond terahertz radiation. Nucl Instrum Methods Phys Res A 2003; 507:537-40.

20. Cole BE, Woodward RM, Crawley D, Wallace VP, Arnone DD, Pepper M. Terahertz imaging and spectroscopy of human skin, in-vivo. Proc SPIE 2001;4276(1-10):1-10.

21. Woodward RM, Cole BE, Wallace VP, Pye RJ, Arnone DD, Linfield EH, et al. Terahertz pulse imaging in reflection geometry of human skin cancer and skin tissue. Phys Med Biol 2002; $47: 3853-63$. 